Journal for ImmunoTherapy of Cancer

\section{Initial dose of oncolytic myxoma virus programs durable antitumor immunity independent of in vivo viral replication}

To cite: Flores EB, Aksoy BA, Bartee E. Initial dose of oncolytic myxoma virus programs durable antitumor immunity independent of in vivo viral replication. Journal for ImmunoTherapy of Cancer 2020;8:e000804. doi:10.1136/ jitc-2020-000804

- Additional material is published online only. To view please visit the journal online (http://dx.doi.org/10.1136/jitc2020-000804).

Accepted 08 May 2020
Check for updates

(c) Author(s) (or their employer(s)) 2020. Re-use permitted under CC BY-NC. No commercial re-use. See rights and permissions. Published by BMJ.

${ }^{1}$ Department of Internal Medicine, Division of Molecular Medicine, University of New Mexico Health Sciences Center, Albuquerque, New Mexico, USA ${ }^{2}$ College of Medicine, Medical University of South Carolina, Charleston, South Carolina, USA

Correspondence to

Dr Eric Bartee;

ebartee@salud.unm.edu

\begin{abstract}
Background Oncolytic therapy uses live-replicating viruses to improve the immunological status of treated tumors. Critically, while these viruses are known to selfamplify in vivo, clinical oncolytic therapies still appear to display a strong dose dependence and the mechanisms mediating this dose dependence are not well understood. Methods To explore this apparent contradiction, we investigated how the initial dose of oncolytic myxoma virus affected the subsequent ability of treatment to alter the immunological status of tumors as well as synergize with programmed cell death protein 1 (PD1) blockade. Results Our results indicate that, due to viral selfamplification in vivo, the overall load of myxoma virus rapidly normalizes within treated tumors despite up to 3-log differences in inoculating dose. Because of this, therapeutic efficacy in the absence of checkpoint blockade is largely dose independent. Despite this rapid normalization, however, treatment with high or low doses of myxoma virus induces distinct immunological changes within treated tumors. Critically, these changes appear to be durably programmed based on the initial oncolytic dose with low-dose treatment failing to induce immunological improvements despite rapidly achieving equivalent viral burdens. Finally, due to the distinct immunological profiles induced by high and low myxoma virus doses, oncolytic efficacy resulting from combination with PD1 blockade therapy displays a strong dose dependence.

Conclusions Taken together, these data suggest that the ability of oncolytic myxoma virus to immunologically reprogram treated tumors is dependent on initial viral dose. Additionally, this work could provide a possible mechanistic explanation for clinical results observed with other oncolytic viruses.
\end{abstract}

\section{INTRODUCTION}

The discovery of T-cell checkpoints, and the subsequent development of clinical checkpoint blockades, have revolutionized cancer treatment. ${ }^{1}$ Unfortunately, while the enthusiasm surrounding checkpointblocking antibodies remains justifiably strong, it is becoming apparent that these therapies require an immunologically "hot" tumor microenvironment due to their dependence on existing antitumor immune responses. ${ }^{23}$ Since many tumors initially present as immunologically "cold," significant interest currently exists around identifying combination therapies involving checkpoint blockade and agents, which can alter the immunological set point of tumors creating a "hot" tumor microenvironment. ${ }^{4}$

Oncolytic virotherapy (OV) represents a form of localized immunotherapy in which cancer-tropic viruses are used to alter the immunological profiles of treated tumors. ${ }^{5}$ One of the major proposed advantages of $\mathrm{OV}$ is the self-amplifying nature of the therapeutic viruses. Theoretically, this self-amplification allows for therapy to be initiated with relatively low inoculating doses which subsequently expand in vivo, increasing the treatment logarithmically. Unfortunately, despite clear evidence that oncolytic viruses do expand during human therapy, a variety of phase I clinical trials (which compare several initial viral doses) have repeatedly demonstrated that efficacy is more often observed following treatment with high doses of virus. ${ }^{6-10}$ Similarly, a phase II dose-finding trial involving the oncolytic vaccinia virus JX594 was stopped prematurely when the early results clearly indicated that higher dose correlated with significantly improved survival. ${ }^{11}$ These results suggest that, in clinical settings, the overall efficacy of OV remains highly dose dependent and no obvious correlation exists between intratumorous viral replication and patient outcomes. ${ }^{6} 711$ To date, however, no clear explanation for this apparent contradiction has been proposed.

We have previously shown that myxoma virus (MYXV)-based OV displays strong synergy with programmed cell death protein 1 (PD1) blockade. ${ }^{12}{ }^{13}$ As translation of this type of combination therapy becomes feasible, however, it is critical to gain a more complete understanding of how therapeutic dose impacts likely outcomes. In the current work, we expand on our previous findings and show that, while viral burden within 
MYXV-treated tumors rapidly normalizes due to in vivo amplification, improvements to the immunological status of treated tumors and therapeutic synergy with PD1 blockade are observed only following high initial oncolytic doses. These data provide a potential mechanistic explanation for the repeated clinical observation that "more oncolytic virus is better."

\section{METHODS}

\section{Cell lines and reagents}

B16/F10 cells (catalog no CRL-6475) were purchased from American Type Culture Collection (Manassas, Virginia, USA). MYXV (strain Lausanne)-expressing GFP from an intergenic region between the m135r and m136r viral open reading frames has been described elsewhere. ${ }^{13}$ The following antibodies were used in these studies: for flow cytometry: CD3 (clone 145-2 c11), CD4 (clone RM4-5), CD8 (clone 53-6.7), CD45 (clone 30-F11), and CD44 (clone IM7) and for PD1 blockade: anti-PD1 (clone RMP1-14) (BioXcell, West Lebanon, New Hampshire, USA).

\section{In vivo tumor models}

C57/B6 mice of 6-8weeks old (Charles River Laboratories, Raleigh, North Carolina, USA) were injected subcutaneously with $4 \times 10^{5} \mathrm{~B} 16 / \mathrm{F} 10$ cells in $50 \mu \mathrm{L}$ of sterile phosphate-buffered saline. Viral treatment consisted of three intratumorous injections of either saline or the indicated dosage of MYXV expressing GFP on days 7, 9, and 11 post-tumor implantation. For efficacy studies, tumor area was monitored using calipers and tumor burden calculated using the formula (tumor area in $\mathrm{mm}^{2}=\mathrm{L} \times \mathrm{W}$ ). For immunological and tumor analysis, tumors were excised, transferred onto a $40 \mu \mathrm{m}$ nylon mesh filter, and mechanically separated into a single cell suspension. Total viral burden was determined by assaying the cellular fraction for infectious particles using standard viral foci forming assays. ${ }^{14}$ TIL were analyzed by staining the cellular fraction for CD45, CD3, CD4, and CD8 using standard methodologies. ${ }^{13}$ All analyses shown are pregated on single, viable events. IFN- $\gamma$ concentration was measured from the soluble fraction of disassociated tumors using the OPTEIA duo ELISA kit (catalog no 551866; BD Biosciences, Franklin Lakes, New Jersey, USA) per manufacturer's recommendations. All experiments were conducted in accordance with the Medical University of South Carolina Institutional Animal Care and Use Committee.

\section{RNAseq and bioinformatics}

For gene expression analysis, tumors were excised on Day 4 after the initiation of treatment and disassociated into single cell suspensions over a $40 \mu \mathrm{m}$ nylon mesh filter. Cells were then pelleted, total RNA extracted using an RNeasy kit (Qiagen, Hilden Germany), and RNAseq performed by Novogene using an Illumina sequencer. Greater than 35M reads were obtained for all samples which were aligned to the mm10 murine reference genome. Principal component analysis was performed using Rstudio and visualized using the ggbiplot package. Unsupervised hierarchical clustering was performed using the edgeR and gplot $\mathrm{R}$ packages.

\section{RESULTS \\ Efficacy of $\mathrm{OV}$ is independent of initial dose in the absence of checkpoint blockade}

To begin to understand the impact of oncolytic dose on therapeutic efficacy, we initially asked how established tumors would respond to injection of differing amounts of oncolytic MYXV. Syngeneic C57/B6 mice were injected subcutaneously with $4 \times 10^{5}$ B16/F10 cells and tumors allowed to establish until they reached $\sim 25 \mathrm{~mm}^{2}$. Tumors were then treated with three injections of either $1 \times 10^{7}, 1 \times 10^{6}$, or $1 \times 10^{5}$ foci-forming units (FFU) of MYXV injected intratumorously (figure $1 \mathrm{~A}$ ) and tumor growth subsequently tracked for 14 days (figure 1B,C). Consistent with previous results, ${ }^{12}{ }^{13}$ treatment of established B16/F10 tumors with $1 \times 10^{7} \mathrm{FFU}$ of MYXV resulted in delayed tumor growth and reduced tumor mass. Interestingly, a similar reduction in tumor growth was observed following injection of either $1 \times 10^{6}$ or $1 \times 10^{5} \mathrm{FFU}$ of MYXV suggesting that, in this context, treatment outcome was independent of initial dose. Consistent with the proposed in vivo self-amplification of oncolytic viruses, analysis of viral burden within tumors also demonstrated that the total amount of infectious particles present 13 days after treatment was similar across all three initial dosages (figure 1D). To further examine the potential normalization of viral burden in vivo, we repeated the experiment above treating tumors with either a high dose of virus $\left(1 \times 10^{6} \mathrm{FFU}\right)$ or a low dose $\left(1 \times 10^{4}\right)$. Tumors were subsequently harvested either 2 or 8 days post-treatment and analyzed for the direct impact of viral infection. Consistent with the 2-log difference in input virus, a significant variance existed between viral burdens in tumors treated with high or low doses 2 days after treatment. This difference, however, rapidly vanished with tumors treated at either dose displaying statistically indistinguishable viral loads by Day 8 (figure 1E). Examination of the number of infected cells revealed a similar phenomenon with tumor sections at Day 8 displaying similar numbers of green flourescent protein $\left(\mathrm{GFP}^{+}\right)$foci (figure $1 \mathrm{~F}$ ) as well as similar numbers of total $\mathrm{GFP}^{+}$cells (figure 1G). Consistent with the previously observed dose-independent delays in tumor growth, both high- and low-dose treatments also caused similar levels of necrotic tissue within treated tumors (figure $1 \mathrm{H}$ ). Taken together, these data support the proposed in vivo amplification of oncolytic viruses and suggest that, in a highly immune suppressive setting where the efficacy of adaptive antitumor immunity is highly blunted, ${ }^{12}{ }^{13}$ treatment outcome is largely independent of initial dose.

\section{OV-based changes to the immunological set point of tumors are dose dependent}

Our previous results suggested that, due to in vivo viral amplification, the impact of direct oncolytic lysis is 
Implant B16/F10 cells subcutaneously
Treat with MYXV

$\left(1 \times 10^{7}, 1 \times 10^{6}\right.$, or $\left.1 \times 10^{5} \mathrm{FFU}\right)$<smiles>O=C(O)C12CC3CC(CC(C3)C1)C2</smiles><smiles>c1ccccc1</smiles>

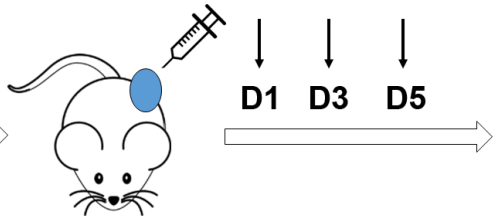

Analyze Day 13

Post-treatment

B

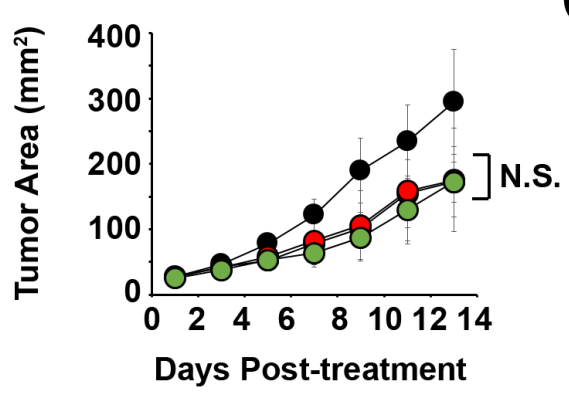

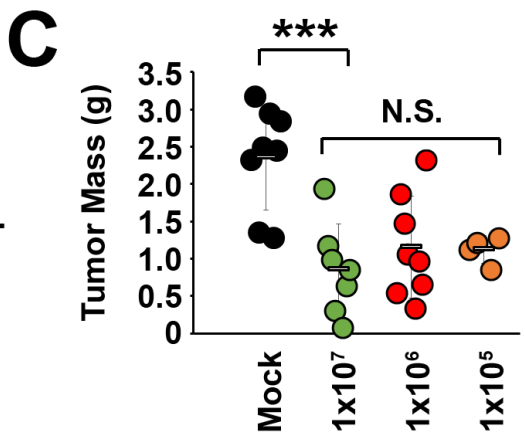

D

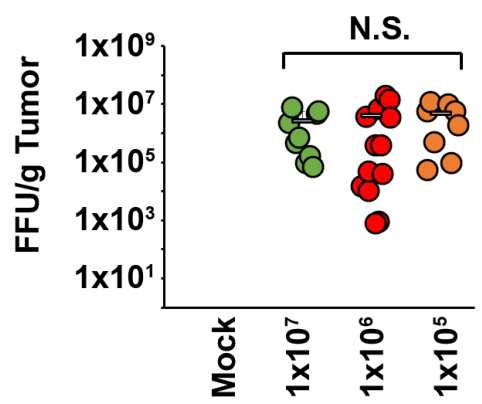

E

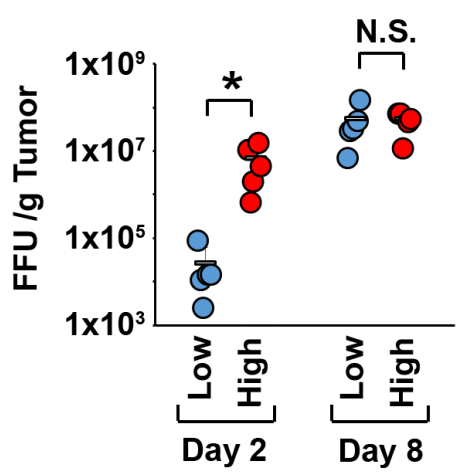

$\mathrm{F}$
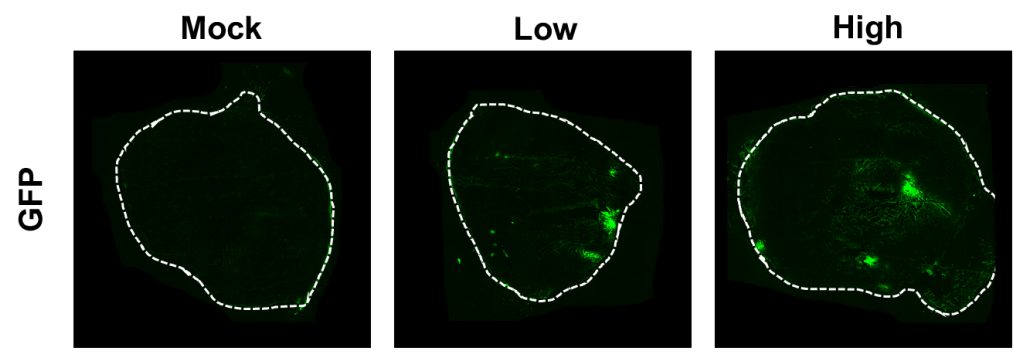

H

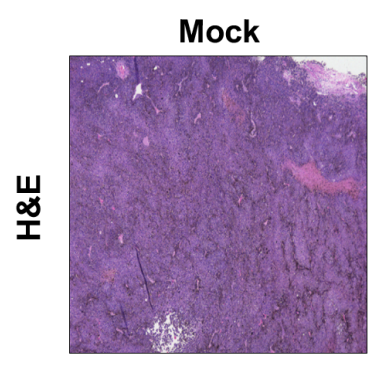

Low

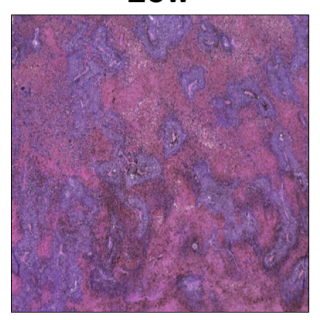

High

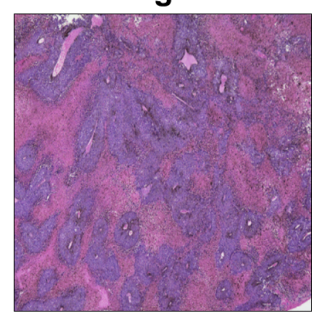

Figure 1 Total load of oncolytic virus normalizes in vivo independent of initial dose. (A) Schematic diagram of experimental design. C57/B6 mice were injected subcutaneously with B16/F10 cells. Once tumors reached $\sim 25 \mathrm{~mm}^{2}$, animals were either mock treated $(n=8)$ or treated with three intratumorous injections of either $1 \times 10^{7}(n=7), 1 \times 10^{6}(n=8)$, or $1 \times 10^{5}(n=8)$ foci forming units (FFU) of myxoma virus (MYXV). (B) Average tumor area $\left(\mathrm{mm}^{2}\right)$ for each treatment over time. (C) Total tumor mass on day 13. (D) Quantitation of infectious virus in each tumor. Data are normalized to tumor mass and displayed as FFU/gram tissue. $(\mathrm{E}-\mathrm{H})$ Tumors were established in mice as above and treated with either a high dose $\left(1 \times 10^{6} \mathrm{FFU}, \mathrm{n}=6\right)$ or a low dose $\left(1 \times 10^{4} \mathrm{FFU}\right.$, $n=6$ ) of MYXV. (E) Quantitation of infectious virus in each tumor either 2 days or 8 days after treatment. Data are normalized to tumor mass and displayed as FFU/gram tissue. (F) Visual depiction of GFP ${ }^{+}$viral foci in snap-frozen tumor sections. (G) Quantitation of the number of GFP ${ }^{+}$tumor cells presents in each tumor at Day 8. $(\mathrm{H})$ Representative images of H\&E-stained tumor sections from each treatment group. Viable tumor is visualized as purple regions while necrotic tumor is visualized as pink. Statistical significance was determined using unpaired Student's t-test (N.S., $\left.{ }^{*} p<0.05,{ }^{* * *} p<0.001\right)$. N.S., not significant. 
independent of initial viral dose. However, human trials have suggested that the primary mechanism responsible for OV's clinical efficacy is modulation of the tumor microenvironment and changing of the immunological set point. We therefore wanted to examine the impact of initial viral dose on the ability of $\mathrm{OV}$ to create an immunologically 'hot' tumor. Syngeneic C57/B6 mice were injected subcutaneously with $4 \times 10^{5}$ B16/F10 cells and tumors allowed to establish until they reached $\sim 25 \mathrm{~mm}^{2}$ and subsequently treated with three injections of either $1 \times 10^{7}, 1 \times 10^{6}, 1 \times 10^{5}$ or $1 \times 10^{4} \mathrm{FFU}$ of MYXV. Tumors were then harvested 4 days after treatment and the overall status of the tumor microenvironment examined using RNAseq. Interestingly, despite our previous results suggesting that varying viral doses resulted in similar delays in tumor growth, principle component analysis of the resulting data sets indicated that tumors treated with high viral doses $\left(1 \times 10^{7}\right.$ or $\left.1 \times 10^{6} \mathrm{FFU}\right)$ displayed highly distinct gene signatures compared with tumors treated with low viral doses $\left(1 \times 10^{5}\right.$ or $\left.1 \times 10^{4} \mathrm{FFU}\right)$ (figure $\left.2 \mathrm{~A}\right)$. This was further supported by unsupervised hierarchical clustering of gene expression which clearly segregated high-dose and lowdose treated tumors (figure 2B). Analysis of the drivers of this segregation revealed both a specific gene signature which did not clearly map to any one cellular pathway as well as clear signatures corresponding to increases in both innate and adaptive immunity (figure 2C). Flow cytometric analysis further confirmed that only treatment with high doses of oncolytic virus increased numbers of $\mathrm{CD}^{+} \mathrm{T}$ cells and total tumor-infiltrating lymphocytes (TILs) (figure 2D). Additionally, only high-dose OV was able to induce expression of the effector molecule interferon- $\gamma($ IFN- $\gamma$ ) (figure 2E). Similar results were observed in the Lewis Lung-A9F1 carcinoma model although in this model the immunological changes were limited to increased $\mathrm{CD}^{+}{ }^{+} \mathrm{T}$-cell numbers (online supplementary figure S1). Taken together these data suggest that, despite rapidly normalizing viral burdens due to in vivo viral amplification, altering the immunological set point of tumors requires treatment with high initial viral doses.

\section{Altering the immunological set point of tumors requires viral replication}

There is currently no real consensus within the field as to whether active viral replication within tumors is required for effective OV. ${ }^{15}$ Multiple groups have shown that inactivated or non-replicative oncolytic virus remains highly effective, ${ }^{16-18}$ while other groups have suggested that inactivation of the virus eliminates its therapeutic efficacy. ${ }^{19-21}$ Since our data suggested that OV-induced changes to the immunological set point of tumors were impacted more by initial oncolytic dose than total viral load, we next asked whether intratumorous replication of virus was required for these changes. Syngeneic C57/B6 mice were injected subcutaneously with $4 \times 10^{5}$ B16/F10 cells and tumors allowed to establish until they reached $\sim 25 \mathrm{~mm}^{2}$. Tumors were then treated with three injections of either a high dose $\left(1 \times 10^{6} \mathrm{FFU}\right)$ or low dose $\left(1 \times 10^{4} \mathrm{FFU}\right)$ of viable or heat-inactivated MYXV (figure 3A) and then analyzed 8 days after the initiation of treatment. Consistent with our previous results (figure 1E), we observed that the total viral load within tumors treated with either high or low doses of viable MYXV completely normalized by 8 days after treatment (figure 3B). Only high-dose treatment, however, resulted in increased numbers of total $\mathrm{CD} 45^{+}$ cells, $\mathrm{CD}^{+}$cells, and IFN- $\gamma$ (figure 3C,D). In contrast, even tumors treated with high doses of heat-inactivated virus showed no increase in immune infiltration or IFN- $\gamma$ levels. These data suggest that, while total viral burden is not predictive of immunological response, some level of productive viral replication is required to induce these changes.

\section{Ineffective initial doses of $\mathrm{OV}$ do not prevent effective secondary treatment with higher doses}

One possible explanation for why low initial doses of OV were ineffective is that suboptimal initial dosing results in the generation of some form of immunological tolerance. This could be achieved either through a true immunological tolerizing response or through low levels of viral infection setting up an active immunological blockade (possibly through secretion of viral immune inhibitors). To test whether either of these possibilities was occurring in our experiments, B16/F10 tumors were established as above and then treated with an initial low dose of MYXV $\left(1 \times 10^{4} \mathrm{FFU}\right)$ followed by two additional injections of a high initial dose $\left(1 \times 10^{6} \mathrm{FFU}\right)$. The standard treatment regimens of three high or three low doses of virus were included as controls (figure 4A). Eight days after the initial low-dose injection, all tumors were harvested and analyzed for viral burden and immunological response. Consistent with our previous results, all treatment regimens displayed statistically indistinguishable total viral loads 8 days after treatment (figure 4B). Additionally, while initial low-dose OV treatments were not sufficient to achieve an immunological response, pretreatment with this ineffective dose did not preclude subsequent immunological responses in either total immune infiltration or IFN- $\gamma$ levels following treatment with a higher dose (figure 4C,D). A minor reduction in CD8+ T-cell infiltration was noted following initial low-dose treatment; however, we attribute this to the slightly reduced time to analysis following high-dose injection (8 days for all highdose treatments versus 6 days for initial low dose, followed by secondary high dose). Taken together, these data suggest that low-dose OV is ineffective due to a failure to reach some necessary threshold and not due to an active immunological blockade.

\section{Initial oncolytic dose results in durable immunological programming}

Our previous results suggested that only high-dose OV altered the immunological set point of tumors at early time points after treatment (figure 2), however, viral burden rapidly normalized following either high-dose or low-dose treatment (figure 1). We therefore wanted to 
A

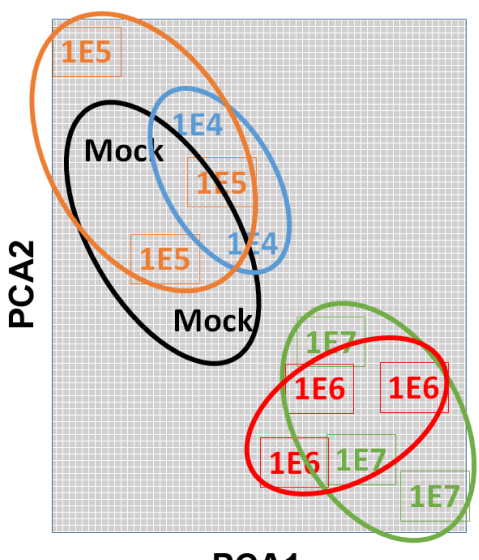

PCA1
B

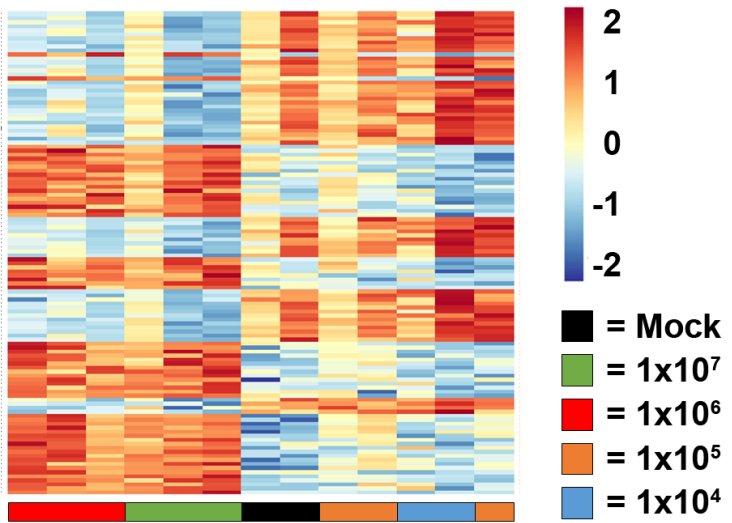

KLH Epha2

Adaptive Immune Signature

\section{Signature}

LARS2

BBC

NPR

NFK

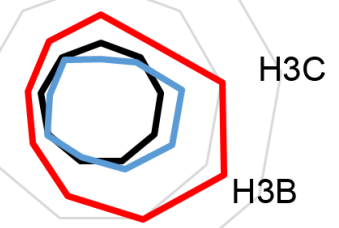

Mock

Mock
$-1 \times 10^{4}$

$-1 \times 10^{6}$

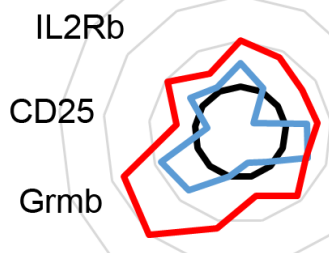

Grma
Innate Immune

Signature CD4

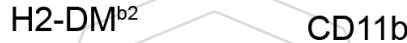

CD3e

CD69

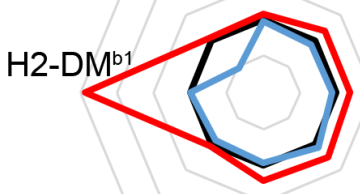

$\mathrm{F} 4 / 80$

IcosL

CD86

CD40

D

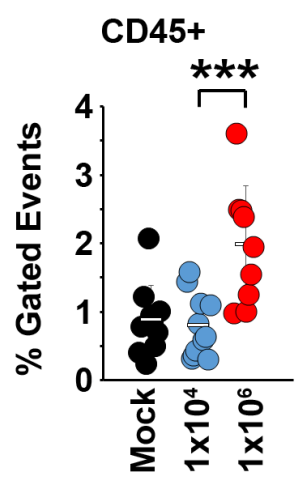

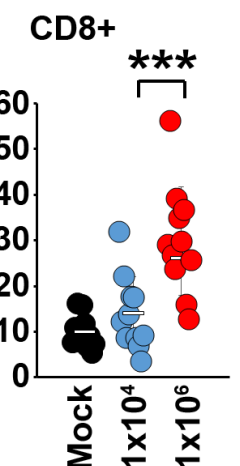

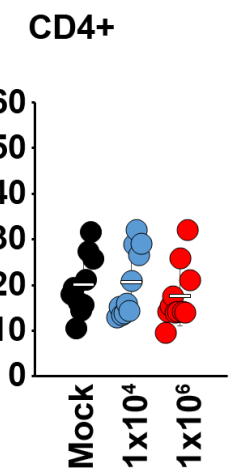

E

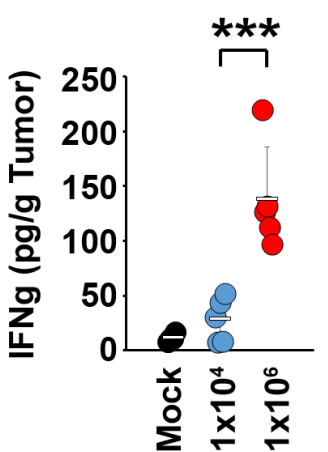

Figure 2 Only high-dose oncolytic therapy improves a tumor's immunological status. Schematic diagram of experimental design. C57/B6 mice were injected subcutaneously with B16/F10 cells. Once tumors reached $\sim 25 \mathrm{~mm}^{2}$, animals were either mock treated or treated with two intratumorous injections of either $1 \times 10^{7}, 1 \times 10^{6}, 1 \times 10^{5}$, or $1 \times 10^{4}$ foci forming units (FFU) of myxoma virus (MYXV) ( $n=3 /$ group). Tumors were harvested 4 days after initiation of treatment and overall gene expression patterns analyzed using RNAseq. (A) Principle component analysis of resulting gene expression profiles indicating treatments cluster into two distinct groups. (B) Hierarchical clustering analysis of gene expression profiles. (C) Visualization of expression profiles from specific gene clusters in mice treated with either $1 \times 10^{6}$ or $1 \times 10^{4} \mathrm{FFU}$ of MYXV. Clusters include a unique gene set made up of 10 most significantly altered genes between the two groups, two curated gene sets made up of known molecules involved in adaptive or innate immunity. (D) Abundance of total $\mathrm{CD} 45^{+}$cells, $\mathrm{CD} 8^{+} \mathrm{T}$ cells, or $\mathrm{CD} 4^{+} \mathrm{T}$ cells within tumors treated as indicated measured by flow cytometry. (E) Abundance of the T-cell effector molecule interferon- $\gamma$ within tumors treated as indicated measured by ELISA. Statistical significance was determined using unpaired Student's t-test $\left({ }^{* \star *} p<0.001\right)$. IFNg, interferon gamma. 

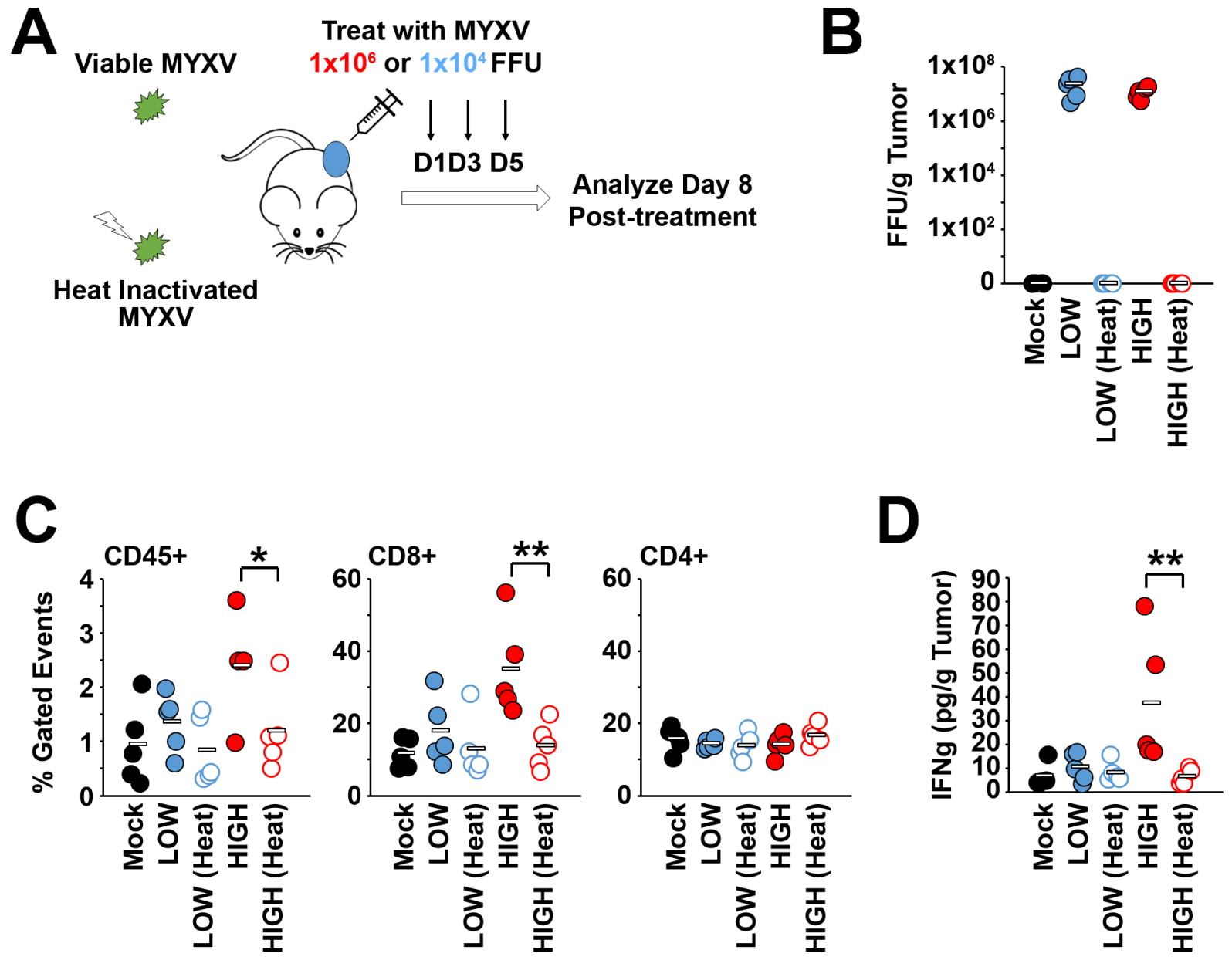

Figure 3 Heat-inactivated mxyoma virus (MXYV) is not sufficient to improve the immunological status of tumors. (A) Schematic diagram of experimental design. C57/B6 mice were injected subcutaneously with B16/F10 cells. Once tumors reached $\sim 25 \mathrm{~mm}^{2}$, animals were either mock treated or treated with three intratumorous injections of either a high dose $\left(1 \times 10^{6}\right.$ foci forming units (FFU)) or low dose $\left(1 \times 10^{4} \mathrm{FFU}\right)$ of live or heat-inactivated MYXV ( $n=5 /$ group). Tumors were harvested 8 days after initiation of treatment for analysis. (B) Quantitation of infectious virus in each tumor. Data are normalized to tumor mass and displayed as FFU/gram tissue. (C) Abundance of total $\mathrm{CD}_{4} 5^{+}$cells, $\mathrm{CD} 8{ }^{+} \mathrm{T}$ cells, or $\mathrm{CD} 4^{+} \mathrm{T}$ cells within tumors treated as indicated measured by flow cytometry. (D) Abundance of the T-cell effector molecule interferon- $\gamma$ within tumors treated as indicated measured by ELISA. Statistical significance was determined using unpaired Student's t-test $\left({ }^{*} \mathrm{p}<0.05,{ }^{* *} \mathrm{p}<0.01\right)$. IFNg, interferon gamma.

know whether treatment with low-dose OV would result in immunological changes after a short delay. Syngeneic C57/B6 mice were injected subcutaneously with $4 \times 10^{5}$ B16/F10 cells and tumors allowed to establish until they reached $\sim 25 \mathrm{~mm}^{2}$. Tumors were then treated with three injections of either a high dose $\left(1 \times 10^{6} \mathrm{FFU}\right)$ or low dose $\left(1 \times 10^{4} \mathrm{FFU}\right)$ of MYXV and subsequently analyzed $2,4,8$, or 12 days after the initiation of treatment (figure 5A). Consistent with our previous results (figure 1E), analysis of infectious particles within treated tumors demonstrated a significant difference immediately following high-dose versus low-dose treatment. This difference, however, disappeared by 4 days post-treatment and viral burden remained statistically indistinguishable at both 8 and 12 days (figure 5B). In contrast to this rapid normalization of viral burden, low-dose OV failed to induce: (1) the previously identified high-dose specific gene signature (figure 5C), (2) a gene signature consistent with alteration of the tumor immunological set point (figure 5D), (3) increased infiltration of either total TIL or $\mathrm{CD}^{+} \mathrm{T}$ cells (figure $5 \mathrm{E}$ ), or (4) increased production of IFN- $\gamma$ (figure 5F) by Day 12 after treatment. These data suggest that, despite normalizations in total viral burden, the immunological responses to OV are durably programmed based on the initial viral dosage.

\section{Therapeutic synergy between OV and PD1 blockade is dose dependent}

We have previously shown that MYXV-based OV displays strong therapeutic synergy with PD1 blockade. ${ }^{12}{ }^{13}$ Since our new data suggested that OV's ability to alter the immunological set point of a tumor is dose dependent, we wanted to expand on our previous studies by analyzing whether the synergy displayed between MYXV and $\alpha-P D 1$ was impacted by initial oncolytic dose. Syngeneic C57/ B6 mice were injected subcutaneously with $4 \times 10^{5} \mathrm{~B} 16 /$ F10 cells and tumors allowed to establish until they reached $\sim 25 \mathrm{~mm}^{2}$. Tumors were then treated with three 

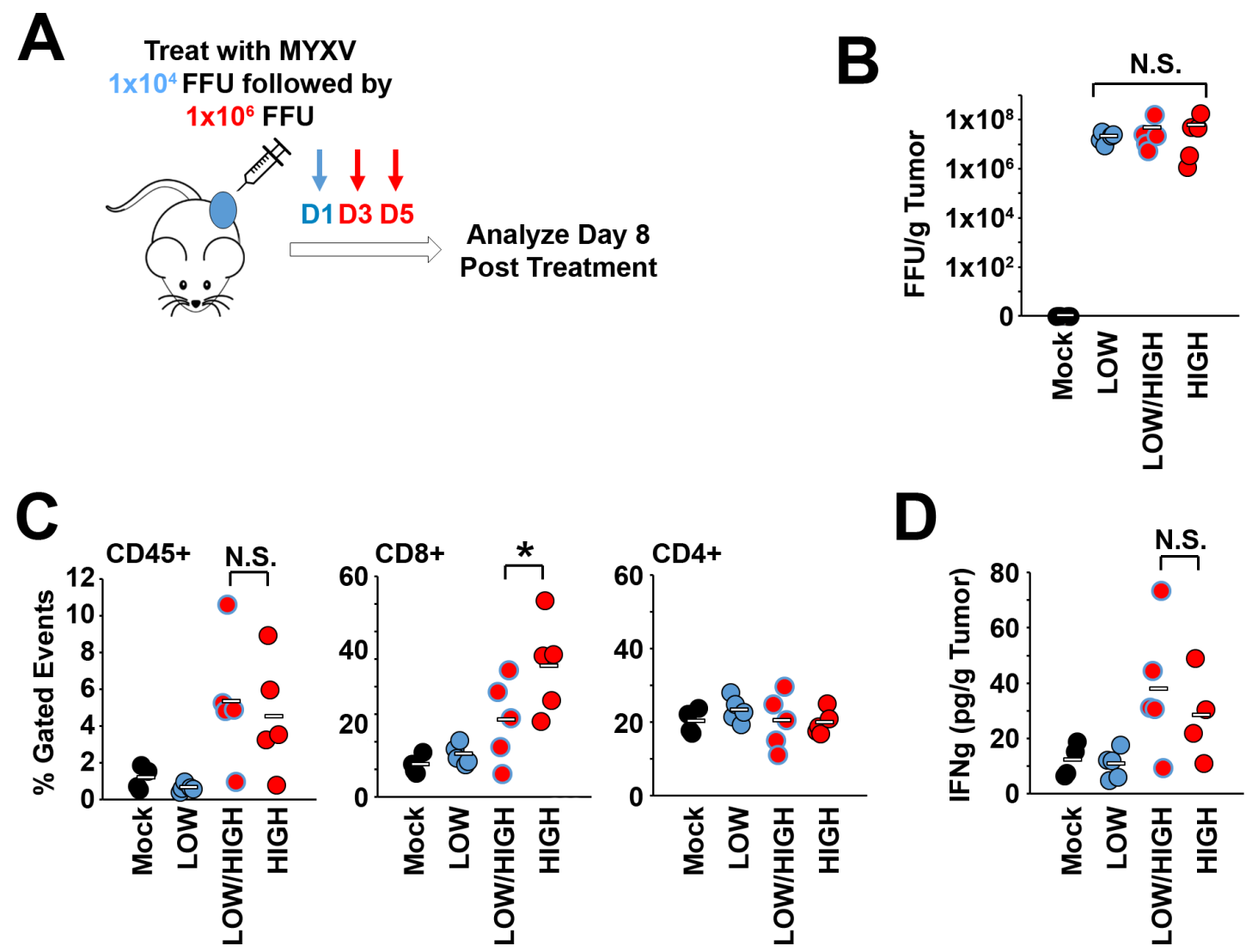

Figure 4 Treatment with low doses of oncolytic virus does not prevent immunological improvement on secondary treatment with high doses. (A) Schematic diagram of experimental design. C57/B6 mice were injected subcutaneously with B16/F10 cells. Once tumors reached $\sim 25 \mathrm{~mm}^{2}$, animals were either mock treated, treated with three intratumorous injections of either a high dose $\left(1 \times 10^{6}\right.$ foci forming units (FFU)) or low dose $\left(1 \times 10^{4} \mathrm{FFU}\right)$ of myxoma virus (MYXV) or treated with a single low dose of virus followed by two secondary treatments with high dose (LOW/HIGH) $(n=5 / g r o u p)$. Tumors were harvested 8 days after initiation of treatment for analysis. (B) Quantitation of infectious virus in each tumor. Data are normalized to tumor mass and displayed as FFU/gram tissue. (C) Abundance of total CD45 ${ }^{+}$cells, $\mathrm{CD}^{+} \mathrm{T}$ cells or CD4 ${ }^{+} \mathrm{T}$ cells within tumors treated as indicated measured by flow cytometry. (D) Abundance of the T-cell effector molecule interferon- $\gamma$ within tumors treated as indicated measured by ELISA. Statistical significance was determined using unpaired Student's t-test $\left({ }^{*} \mathrm{p}<0.05\right.$, N.S.). IFNg, interferon gamma; N.S., not significant.

injections of $1 \times 10^{7}, 1 \times 10^{6}$ or $1 \times 10^{5} \mathrm{FFU}$ of MYXV as well as intraperitoneal injections of PD1-blocking antibody (figure 6A). Tumor burden was then monitored every other day for 18 days to determine therapeutic efficacy. The results indicated that mice treated with MYXV and $\alpha \mathrm{PD} 1$ displayed delayed tumor growth (figure $6 \mathrm{~B}$ ) and significantly reduced tumor burden at Day 11 (figure 6C) compared with mock-treated controls as well as prolonged overall survival (online supplementary figure S2). Interestingly, unlike MYXV-based treatment in the absence of PD1 blockade (figure 1), delays in tumor growth now displayed a strong dose dependence with higher initial doses of virus resulting in improved therapeutic outcomes. A similar dose dependence was observed in a B16/F10 model, which is inherently susceptible to MYXV therapy due to genetic deficiency in programmed death ligand 1 expression (figure 6D-F, online supplementary figure S3).

\section{Discussion}

One of the major proposed advantages of OV is the selfamplification of the therapeutic viruses. This amplification theoretically allows for treatment to be initiated by relatively low doses of virus which subsequently expand in vivo enhancing the therapeutic efficacy. In contrast to this theory, human trials have demonstrated that the clinical application of OV displays a relatively strong dose dependence with more virus typically being associated with better outcomes. ${ }^{6-11}$ Here we show that the total viral burden within treated tumors rapidly normalizes across at least 3 logs regardless of initial oncolytic dose (figures 1-4). In striking contrast to this normalization, however, the ability of OV to induce changes to a tumor's immunological profile displays strong dose dependence. High doses $\left(>1 \times 10^{6} \mathrm{FFU}\right.$ in our experiments) were able to induce substantial changes to overall gene expression within the tumor microenvironment including significant increases in total TIL and $\mathrm{CD}^{+} \mathrm{T}$ cells which are likely essential for therapeutic success (figure 2). Critically, these changes could not be observed following low-dose therapy $\left(<1 \times 10^{6} \mathrm{FFU}\right)$ even 8 days after viral burden appeared to normalize (figure 5). Interestingly, this cut-off appeared quite distinct in our experiments 


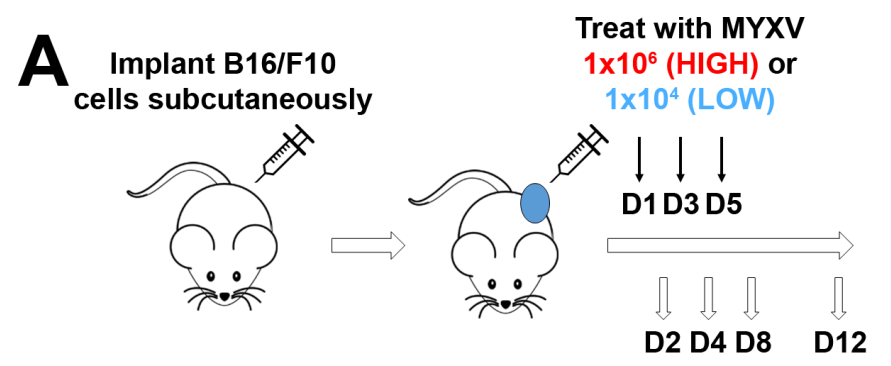

Harvest for analysis

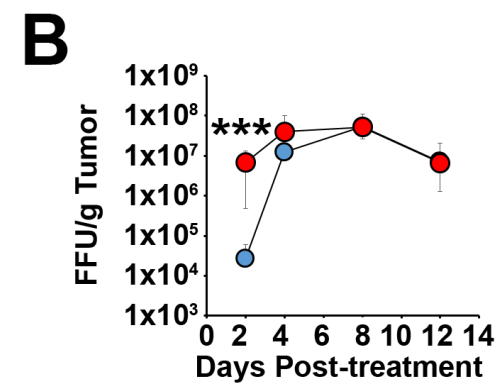

Day 12
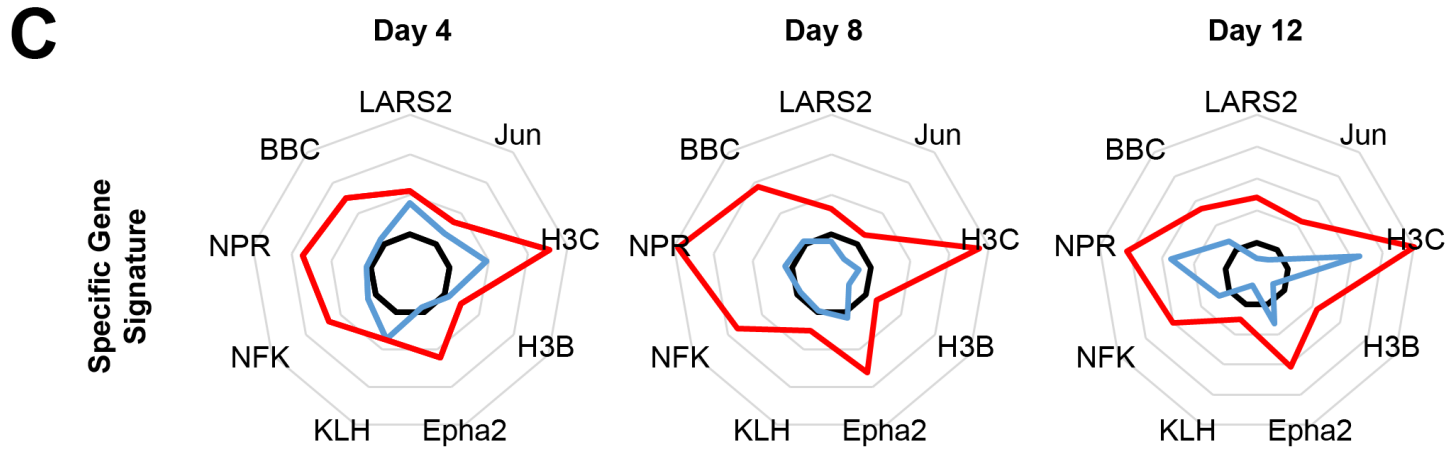

D

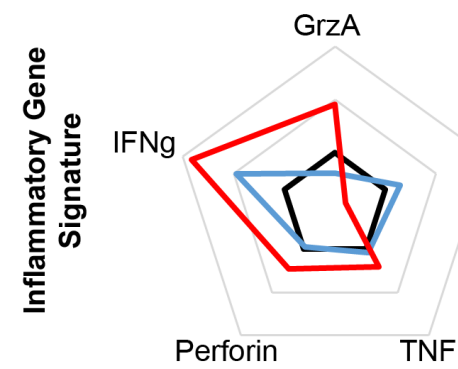

GrzB

IFNg

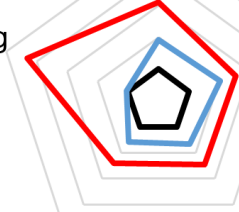

GrzB

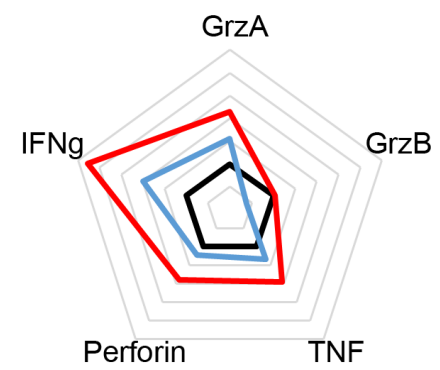

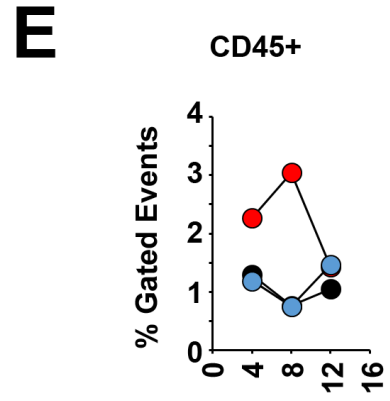

CD8+

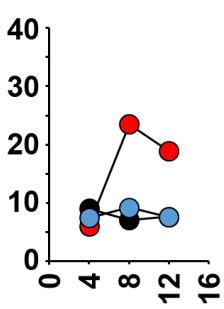

Days Post-treatment
CD4+

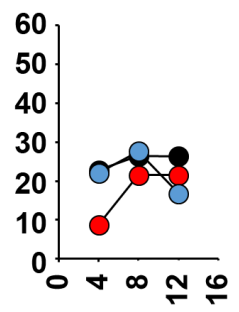

\section{$\stackrel{2}{2}$}

.

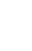

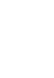

Figure 5 Initial oncolytic dose determines a durable immunological program. (A) Schematic diagram of experimental design. C57/B6 mice were injected subcutaneously with B16/F10 cells. Once tumors reached $\sim 25 \mathrm{~mm}^{2}$, animals were either mock treated or treated with three intratumorous injections of either a high $\left(1 \times 10^{6}\right.$ foci forming units (FFU)) or low $\left(1 \times 10^{4} \mathrm{FFU}\right)$ oncolytic dose of myxoma virus (MYXV). Tumors were then harvested 2, 4, 8, or 12 days after the initiation of treatment ( $\mathrm{n}=5 /$ time point/ dose). (B) Quantitation of infectious virus in each tumor at each time point. Data are normalized to tumor mass and displayed as FFU/gram tissue. (C) Expression of the top 10 most significantly altered genes 4, 8, and 12 days post-treatment measured by quantitative-polylmerase chain reaction (qt-PCR). (D) Expression of a curated gene set made up of known adaptive immune mediators 4, 8 , and 12 days post-treatment measured by qt-PCR. (E) Abundance of total CD45 $5^{+}$cells, $C D 8^{+} \mathrm{T}$ cells, or $\mathrm{CD} 4^{+} \mathrm{T}$ cells within tumors treated as indicated at 4,8 , or 12 days post-treatment measured by flow cytometry. (E) Abundance of the T-cell effector molecule interferon- $\gamma$ within tumors treated as indicated at 4,8 , or 12 days post-treatment measured by ELISA. Statistical significance was determined using unpaired Student's t-test $\left.{ }^{* \star *} \mathrm{p}<0.001\right)$. IFNg, interferon gamma. 
A

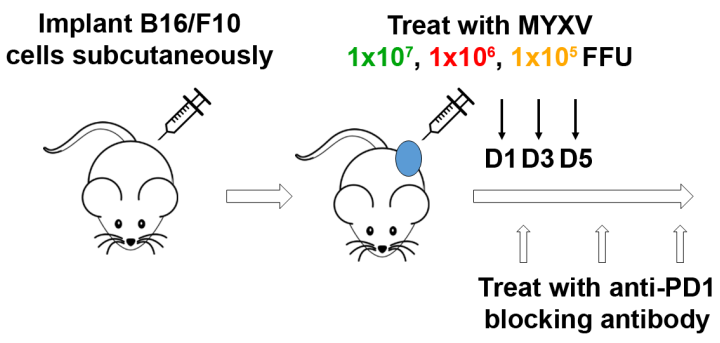

D

Implant B16/F10-PDL1 ${ }^{-1}$ cells subcutaneously

Treat with MYXV
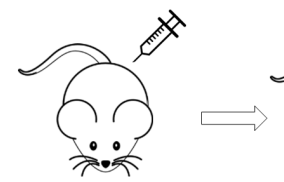

$1 \times 10^{7}, 1 \times 10^{6}, 1 \times 10^{5} \mathrm{FFU}$

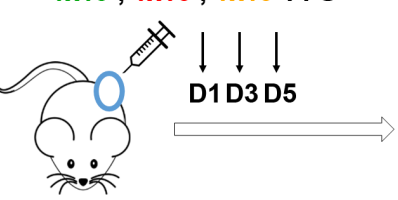

B

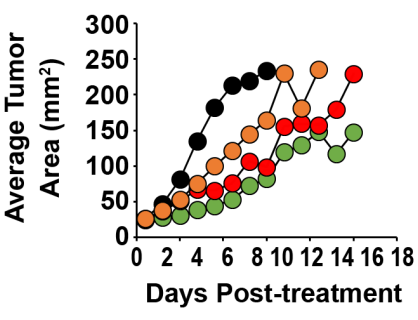

C

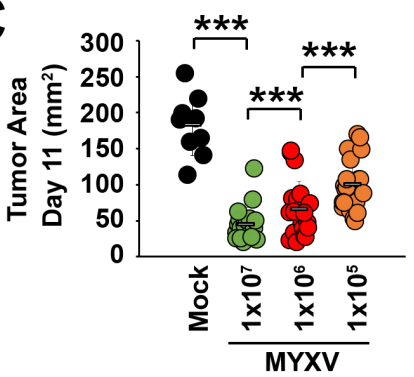

E

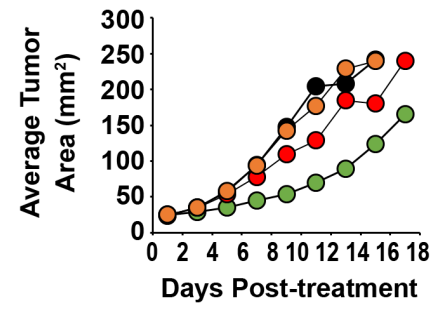

Figure 6 Efficacy of combined oncolytic virotherapy/programmed cell death protein 1 (PD1) blockade therapy is dose dependent. (A) Schematic diagram of experimental design. C57/B6 mice were injected subcutaneously with B16/F10 cells. Once tumors reached $25 \mathrm{~mm}^{2}$, animals were either mock treated or treated with three intratumorous injections of $1 \times 10^{7}, 1 \times 10^{6}$, or $1 \times 10^{5}$ foci forming units (FFU) of myxoma virus (MYXV) ( $n=10+/$ group). Animals were subsequently given intraperitoneal injections of PD1-blocking antibody and monitored for tumor burden every other day. (B) Average tumor area $\left(\mathrm{mm}^{2}\right)$ for each treatment over time. (C) Total tumor area on day 11. (D) Schematic diagram of experimental design. C57/B6 mice were injected subcutaneously with B16/F10-PDL $1^{-/-}$cells. ${ }^{12}$ Once tumors reached $\sim 25 \mathrm{~mm}^{2}$, animals were either mock treated or treated with three intratumorous injections of $1 \times 10^{7}, 1 \times 10^{6}$, or $1 \times 10^{5} \mathrm{FFU}$ of MYXV ( $\mathrm{n}=7+$ /group) and monitored for tumor burden every other day. (E) Average tumor area $\left(\mathrm{mm}^{2}\right)$ for each treatment over time. (F) Total tumor area on day 11. Statistical significance was determined using unpaired Student's t-test $\left(^{\star \star \star} \mathrm{p}<0.001\right)$. PDL1, programmed death-ligand 1.

with tumorous gene expression profiles clustering into "hot" and "cold" groups as opposed to a more linear dose dependence (figure 2A,B). This result suggests that some threshold might exist which determines the ability of OV to induce inflammation. Oncolytic doses below this threshold would likely fail to improve immunological status, while oncolytic doses above the threshold would be successful. While our studies identify this threshold as $1 \times 10^{6} \mathrm{FFU}$ in our model, it is unlikely that this number represents the required oncolytic dose for all tumors. Additional work is therefore needed to identify the various factors which might influence the dose required to alter the immunological status of a given tumor. There are likely numerous such factors; however, a few obvious candidates might include tumor size, tumor location, existing immunological status, and susceptibility of tumor to initial viral infection.

Critically, while in vivo viral amplification following low-dose therapy did not appear to induce improvements in immunological status, some form of viral replication was required since even high-dose treatment with heatinactivated MYXV failed to alter a tumor's immune set point (figure 3). Additionally, while initial treatment with low-dose OV failed to induce immunological improvements, it also did not appear to preclude these improvements following a secondary rescue treatment with high-dose OV (figure 4). This is consistent with limited results in human patients treated with Imlygic who, having failed a first course of treatment, subsequently responded to an identical second course (K Shirai, personal communication). These data suggest that any potential therapeutic threshold which exists might be fluid with the effective oncolytic dose required to achieve immunological improvements potentially changing over time.

Finally, it should be noted that our results were obtained using oncolytic MYXV. Unlike most oncolytic viruses, MYXV displays relatively slow rates of spread in vivo due to its failure to produce significant amounts of extracellular-free virions. This is clearly observed in our current results since the maximum rate of infection seen in tumors treated with even high doses of MYXV remains $4 \%$ (figure 1). It is therefore possible that lower doses of oncolytic viruses which display more rapid spread (such as measles or vesicular stomatitis virus) might be more readily able to alter the immunological set point of tumors. Alternatively, achieving an immunological response with these viruses could also display an initial dose dependence; however, the threshold required to reach this might be lower due to the higher rate of viral spread in vivo. In this regard, it would be interesting to compare the effective oncolytic dose of various viruses with each virus's in vivo spread to determine whether more rapid viral spread (as opposed to high intracellular viral burden) allowed for reduced initial dose. In 
contrast, each oncolytic virus contains its own series of pathogen associated molecular pattern (PAMPs) as well as encoding its own immune modulatory repertoire. The intrinsic biological properties of each oncolytic virus could therefore play a significant role in its ability to alter the tumor immune profile at various dosages. Critically, little work has been done comparing various oncolytic platforms to each other in vivo. Whether the inherent inflammatory properties of each virus impact OV's ability to induce intratumorous inflammation therefore remains poorly understood.

Acknowledgements We thank Dr Chrystal Paulos for valuable discussion of this project.

Contributors EF conducted all experiments, collected, and analyzed data. BAA performed bioinformatics analysis. EB designed and analyzed experiments, oversaw project, and prepared manuscript.

Funding This work was supported by grants to Dr Eric Bartee from: NIH-NCI (R01-CA194090), NIH-NIAID (R21-Al142387), the ACS (RSG-17-047-01-MPC), and intramural funding from both the Medical University of South Carolina and the University of New Mexico Health Sciences Center. This work was also supported in part by the Hollings Cancer Center's Support Grant (P30-CA138313).

Competing interests None declared.

Patient consent for publication Not required.

Provenance and peer review Not commissioned; externally peer reviewed.

Data availability statement Data are available on reasonable request. The only largeish dataset in the current work is a RNAseq dataset, which is available to anyone on request.

Open access This is an open access article distributed in accordance with the Creative Commons Attribution Non Commercial (CC BY-NC 4.0) license, which permits others to distribute, remix, adapt, build upon this work non-commercially, and license their derivative works on different terms, provided the original work is properly cited, appropriate credit is given, any changes made indicated, and the use is non-commercial. See http://creativecommons.org/licenses/by-nc/4.0/.

\section{ORCID iD}

Eric Bartee http://orcid.org/0000-0003-1793-446X

\section{REFERENCES}

1 Couzin-Frankel J. Breakthrough of the year 2013. cancer immunotherapy. Science 2013;342:1432-3.

2 Tomioka N, Azuma M, Ikarashi M, et al. The therapeutic candidate for immune checkpoint inhibitors elucidated by the status of tumorinfiltrating lymphocytes (TILs) and programmed death ligand 1 (PDL1) expression in triple negative breast cancer (TNBC). Breast Cancer 2018;25:34-42.

3 Yagi T, Baba Y, Ishimoto T, et al. PD-L1 expression, tumor-infiltrating lymphocytes, and clinical outcome in patients with surgically resected esophageal cancer. Ann Surg 2019;269:471-8.
4 Popovic A, Jaffee EM, Zaidi N. Emerging strategies for combination checkpoint modulators in cancer immunotherapy. J Clin Invest 2018;128:3209-18.

5 Melcher A, Parato K, Rooney CM, et al. Thunder and lightning: immunotherapy and oncolytic viruses collide. Mol Ther 2011;19:1008-16.

6 Dispenzieri A, Tong C, LaPlant B, et al. Phase I trial of systemic administration of Edmonston strain of measles virus genetically engineered to express the sodium iodide symporter in patients with recurrent or refractory multiple myeloma. Leukemia 2017;31:2791-8.

7 Machiels J-P, Salazar R, Rottey S, et al. A phase 1 dose escalation study of the oncolytic adenovirus enadenotucirev, administered intravenously to patients with epithelial solid tumors (evolve). $J$ Immunother Cancer 2019;7:20.

8 Park SH, Breitbach CJ, Lee J, et al. Phase 1B trial of biweekly intravenous Pexa-Vec (JX-594), an oncolytic and immunotherapeutic vaccinia virus in colorectal cancer. Mol Ther 2015;23:1532-40.

$9 \mathrm{Kim} \mathrm{KH}$, Dmitriev IP, Saddekni S, et al. A phase I clinical trial of Ad5/3- $\Delta 24$, a novel serotype-chimeric, infectivity-enhanced, conditionally-replicative adenovirus (CRAd), in patients with recurrent ovarian cancer. Gynecol Oncol 2013;130:518-24.

10 Chiocca EA, Abbed KM, Tatter S, et al. A phase I open-label, dose-escalation, multi-institutional trial of injection with an E1BAttenuated adenovirus, ONYX-015, into the peritumoral region of recurrent malignant gliomas, in the adjuvant setting. Mol Ther 2004;10:958-66.

11 Heo J, Reid T, Ruo L, et al. Randomized dose-finding clinical trial of oncolytic immunotherapeutic vaccinia JX-594 in liver cancer. Nat Med 2013;19): :329-36.

12 Bartee MY, Dryja PC, Bartee E. Chimeric tumor modeling reveals role of partial PDL1 expression in resistance to virally induced immunotherapy. J Immunother Cancer 2019;7:11.

13 Bartee MY, Dunlap KM, Bartee E. Tumor-Localized secretion of soluble PD1 enhances oncolytic virotherapy. Cancer Res 2017;77:2952-63.

14 Smallwood SE, Rahman MM, Smith DW, et al. Myxoma virus: propagation, purification, quantification, and storage. Curr Protoc Microbiol 2010; Chapter 14:1-14.

15 Davola ME, Mossman KL. Oncolytic viruses: how "Iytic" must they be for therapeutic efficacy? Oncoimmunology 2019;8:e1581528.

16 Galivo F, Diaz RM, Wongthida P, et al. Single-Cycle viral gene expression, rather than progressive replication and oncolysis, is required for VSV therapy of B16 melanoma. Gene Ther 2010;17:158-70.

17 Dai P, Wang W, Yang N, et al. Intratumoral delivery of inactivated modified vaccinia virus Ankara (iMVA) induces systemic antitumor immunity via STING and Batf3-dependent dendritic cells. Sci Immunol 2017;2. doi:10.1126/sciimmunol.aal1713. [Epub ahead of print: 19 May 2017].

18 Prestwich RJ, llett EJ, Errington F, et al. Immune-Mediated antitumor activity of reovirus is required for therapy and is independent of direct viral oncolysis and replication. Clin Cancer Res 2009;15:4374-81.

19 Wongthida P, Diaz RM, Galivo F, et al. Vsv oncolytic virotherapy in the B16 model depends upon intact MyD88 signaling. Mol Ther 2011;19:150-8.

20 Wongthida P, Diaz RM, Galivo F, et al. Type III IFN interleukin-28 mediates the antitumor efficacy of oncolytic virus VSV in immunecompetent mouse models of cancer. Cancer Res 2010;70:4539-49.

$21 \mathrm{Wu}$ Y, Lun X, Zhou H, et al. Oncolytic efficacy of recombinant vesicular stomatitis virus and myxoma virus in experimental models of rhabdoid tumors. Clin Cancer Res 2008;14:1218-27. 